Article

\title{
Genome-Wide Analysis of Auxin Receptor Family Genes in Brassica juncea var. tumida
}

\author{
Zhaoming Cai ${ }^{1}$, De-er Zeng ${ }^{2}$, Jingjing Liao ${ }^{1}$, Chunhong Cheng ${ }^{1}$, Zulfiqar Ali Sahito ${ }^{3}$, \\ Meiqin Xiang ${ }^{1}$, Min Fu ${ }^{1}$, Yuanqing Chen ${ }^{1}$ and Diandong Wang ${ }^{1, *}$ \\ 1 College of Life Science and Technology, Yangtze Normal University, Chongqing 408100, China; \\ caizhaoming-2000@163.com (Z.C.); 10091123@163.com (J.L.); xiaobei15109217512@163.com (C.C.); \\ 18323997970@163.com (M.X.); FUMIN55555@126.com (M.F.); 17729647355@163.com (Y.C.) \\ 2 School of Life Sciences, Provincial Key Laboratory of the Biodiversity Study and Ecology Conservation in \\ Southwest Anhui, Anqing Normal University, Anqing 246133, China; qiuyizeng@163.com \\ 3 State Key Laboratory of Plant Cell and Chromosome Engineering, Institute of Genetic and Developmental \\ Biology, Chinese Academy of Sciences, Beijing 100101, China; sahito_zulfiqarali@yahoo.com \\ * Correspondence: wangdiandongyznu@163.com; Tel.: +86-15736563457
}

Received: 23 January 2019; Accepted: 18 February 2019; Published: 20 February 2019

\begin{abstract}
Transport inhibitor response 1/auxin signaling f-box proteins (TIR1/AFBs) play important roles in the process of plant growth and development as auxin receptors. To date, no information has been available about the characteristics of the TIR1/AFB gene family in Brassica juncea var. tumida. In this study, 18 TIR1/AFB genes were identified and could be clustered into six groups. The genes are located in 11 of 18 chromosomes in the genome of B. juncea var. tumida, and similar gene structures are found for each of those genes. Several cis-elements related to plant response to phytohormones, biotic stresses, and abiotic stresses are found in the promoter of BjuTIR1/AFB genes. The results of qPCR analysis show that most genes have differential patterns of expression among six tissues, with the expression levels of some of the genes repressed by salt stress treatment. Some of the genes are also responsive to pathogen Plasmodiophora brassicae treatment. This study provides valuable information for further studies as to the role of BjuTIR1/AFB genes in the regulation of plant growth, development, and response to abiotic stress.
\end{abstract}

Keywords: Brassica juncea var. tumida; auxin receptor; TIR1; AFB; gene expression pattern; abiotic stress

\section{Introduction}

Auxin is one of most important phytohormones, playing a crucial role in most physiological and biochemical processes of plant growth and development, such as embryo and fruit development, tissue differentiation, root initiation, plant gravitropism, and plant response to biotic and abiotic stresses [1-4]. The transport inhibitor response 1/auxin signaling f-box protein (TIR1/AFB) family genes perceive the auxin signal as auxin receptors, which then trigger the auxin response signaling pathway $[5,6]$. It has been reported that TIR1 and the AFBs are located in the nucleus and bind auxin [5,6]; the nuclear localization of GmTIR1 and GmAFB3 was also reported in soybean [7]. After auxin molecules bind to TIR1/AFB proteins, the activated TIR1/AFB proteins react with auxin/indole-3-acetic acid (Aux/IAA) family proteins [8]. The $A u x / I A A$ family genes play important roles in the regulation of auxin response factor genes $(A R F s)$. These $A R F$ family genes are notable for their ability to directly activate or inhibit auxin responsive genes at the transcription level [9-11]. This TIR1/AFB-Aux/IAA-ARF cascade comprises a typical auxin signaling pathway.

At conditions of low auxin concentration in the plant, the Aux/IAA proteins could bind to ARFs, which results in the inhibition of the transcriptional activity of ARFs. When auxin molecules accumulate 
in plant cells, the reaction between the auxin molecules and TIR1/AFBs triggers a combination of TIR1/AFBs and Aux/IAAs, leading to degradation of the Aux/IAA proteins through the ubiquitin-26S proteasome system. After the degradation of Aux/IAA proteins, the released ARFs could effectively activate a form of auxin response system gene, which attempts to correctly regulate the plant's response to auxin signaling [12].

TIR1 was the first identified in Arabidopsis, which includes an F-box domain in the $\mathrm{N}$ terminus and several Leucine-rich repeat (LRR) domains in the $C$ terminus [13]. The tir1 loss-of-function mutants are deficient in some auxin-regulated growth processes, such as hypocotyl elongation and lateral root formation [13]. Overexpression of AtTIR1 results in inhibited primary root growth, agravitropic root tips, and promoted lateral root development [14]. The TIR1 functions as the substrate receptor of SCF E3 complex, which could specially degrade Aux/IAA proteins through the ubiquitin-26S proteasome system. Auxin molecules could promote the interaction between the Aux/IAA and SCF ${ }^{\mathrm{TIR} 1}$ complex by binding directly to SCF ${ }^{\mathrm{TIR} 1}$; TIR1 is identified as an auxin receptor [5,6]. Shortly after that, three genes named $A F B 1, A F B 2$, and $A F B 3$ were identified, playing similar roles to TIR1 in the same manner [5]. It is worth noting that while all the four genes TIR1, AFB1, AFB2m and AFB3 play roles in the plant response to auxin in the root, TIR1, AFB2, and AFB3 contribute equally in this process, while AFB1 does not assemble into an SCF complex as efficiently [5,15]. For the phenotype of pant growth and development, the single mutant of $a f b 1$, afb2, and $a f b 3$ seedlings all performed with a similar phenotype to the wild type, even in double mutant combinations involving the $a f b$ mutants and tir1; no obvious development defects have been found. However, severe development defects in cotyledon, hypocotyl elongation, and gravitropism have been observed in triple and quadruple mutants [5]. This suggests that those four genes have redundant functions during plant growth and development. It has been reported that both AFB4 and AFB5 function as auxin receptors. No obvious defect is found on growth of the seedlings with afb4 loss-of-function mutants [16]. Both $a f b 4$ and $a f b 5$ are resistant to the synthetic auxin picloram but not IAA or 2,4-D, indicating that these two proteins are selective for picloram [16-18].

IAA has been found to induce main root growth inhibition rapidly, while the TIR1/AFB's auxin receptor mutants failed to display this effect $[19,20]$. In Arabidopsis thaliana, overexpression of AtTIR1 has resulted in overproduction of lateral roots [21]. Similarly, knocking down GmTIR1 and GmAFB3 genes resulted in fewer nodule numbers in soybean [7]. It has been reported that AtAFB3 plays important roles in lateral root production, in the manner of specially responding to nitrate treatment [22]. In the $a f b 4$ loss-of-function mutant, the number of lateral roots is significantly less than that in wild-type seedlings, indicating that AFB4 is also involved in lateral root development in Arabidopsis [23]. Besides that, the afb4 mutant had other defects in the development process, such as shorter hypocotyls, lower height, and delayed flowering [23]. The TIR1/AFB-mediated auxin signaling pathway is also involved in plant tolerance to abiotic or biotic stresses. In Arabidopsis, lateral root production is normally inhibited by ABA treatment-however, overexpression of AtAFB2 has been shown to counteract this defect, indicating a response pathway from abiotic stress to this particular gene [24]. In Arabidopsis, the transcriptional levels of AtTIR1, AtAFB1, AtAFB2, and AtAFB3 are reduced by a flagellin-derived, 22-amino acid peptide (flg22, bacterial pathogen attack), resulting in the repression of auxin signaling in the plant. Repressed auxin sensitivity could restrict growth of pathogens, further highlighting the crucial roles of TIR1/AFBs in plant tolerance to abiotic stresses [25].

Tumorous stem mustard (Brassica juncea var. tumida Tsen et Lee) is an important crop in China, with great economic benefits. How to improve the yield of this crop is a key issue for the Chinese pickle industry. Auxin plays pleotropic roles in plant growth and development, exploring whether or not the effect of the auxin signaling pathway in crop production is meaningful. TIR1/AFB receptors also exist in tumorous stem mustard; however, no related report has been found for the function of those genes in this crop. Here, we have identified the TIR1/AFB family members in tumorous stem mustard, and analyzed their genes and the protein characteristics of those genes, as well as their expression 
patterns during plant growth and development. This study will be useful as a starting point for more functional investigations of TIR1/AFBs in tumorous stem mustard.

\section{Materials and Methods}

\subsection{Plant Materials and Growth Conditions}

In this study, tumorous stem mustard cultivar Yong An XiaoYe was used for gene expression pattern analysis. The seeds were sowed into 2:1 vermiculite:turfy soil, cultured under constant light at $22{ }^{\circ} \mathrm{C}$ with a $16 / 8 \mathrm{~h} \mathrm{light/dark}$ regime in culture room. For the salt stress assay, the seeds of Yong An Xiao Ye were sowed in pots in a culture room, and two-week-old seedlings were irrigated by nutrient solution with or without $200 \mathrm{mM} \mathrm{NaCl}$ for 3, 6, 12, $24 \mathrm{~h}$, respectively, and the roots were collected for real-time quantitative PCR analysis. For pathogen treatment, two-week-old seedlings were then inoculated with a $5 \mathrm{~mL}$ resting spore suspension of Plasmodiophora brassicae $\left(\mathrm{OD}_{600}=0.07\right)$ for the indicated time points.

2.2. The Identifications of Transport Inhibitor Response 1/Auxin Signaling F-Box Protein in Tumorous Stem Mustard and Phylogenetic Analysis

The TIR1, AFB1, AFB2, AFB3, AFB4, and AFB5 sequence of $A$. thaliana were obtained from The Arabidopsis Information Resource website (https://www.arabidopsis.org/). Using the TIR1/AFB genes as the query sequence to blast relative homologs in the B. juncea genome database (http:/ /brassicadb. $\mathrm{org} / \mathrm{brad} /$ ). The gene structure diagram was drawn using the online software of the GSDS2.0 server (http:/ /gsds.cbi.pku.edu.cn/), the phylogenetic analysis was done using MEGA5 software [26] with the neighbor-joining method. The bootstrap value was 1000 replicates.

\subsection{The Analysis of Protein Structures and Characters}

The amino acid isoelectric point and molecular weight were predicted by online ExPASy software (https://web.expasy.org/compute_pi/), and the protein secondary structure was analyzed by SMART (http://smart.embl-heidelberg.de/) and CDD (https://www.ncbi.nlm.nih.gov/Structure/cdd/wrpsb. cgi). The diagram of the protein domain was drawn by ExPASy. The cis-elements in the promotor of TIR1/AFBs were predicted by SOGO online software (https:/ / sogo.dna.affrc.go.jp/cgi-bin/sogo.cgi? lang $=$ en\&pj $=640 \&$ action $=$ page \&page $=$ newplace).

\subsection{RNA Extraction and Real-Time Quantitative PCR Analysis}

The total RNA was extracted from different plant materials using Trizol reagent (Tiangen Biotech Co., Ltd., Beijing, China). The total RNA samples were treated with DNase I (Invitrogen, Carlsbad, CA, United States) to remove contaminating genomic DNA. First-strand cDNA was synthesized from the total RNA using a FastQuant RT Kit (Tiangen Biotech (Beijing) Co., Ltd.). Real-time qPCR (qRT-PCR) was performed using SuperReal PreMix Plus (SYBR Green; Tiangen Biotech (Beijing) Co., Ltd.). BjuACTIN3 was used as the internal reference gene for qRT-PCR [27], and the gene-specific primers are listed in Supplementary Table S1.

\subsection{Statistical Analysis}

All data were analyzed using SigmaPlot 10.0 (Systat Software, Inc., Chicago, IL, United States) and SPSS 16.0 software. The averages and standard deviations of all results were calculated, one-way ANOVA followed by the Dunnett test was used. 


\section{Results}

\subsection{The Identification of TIR1/AFB Homologs in Brassica juncea var. tumida}

Eighteen genes were identified as homologs of TIR1/AFB genes in B. juncea var. tumida; they were named BjuTIR1 and BjuAFBs, and the CDS sequences of those genes can be found in Supplementary File S2. The open reading frame of BjuTIR1/AFBs varied between 1566 bp to $1830 \mathrm{bp}$, coding 521 to 609 amino acids; the molecular weight was 58.58-68.13 and the isoelectric point (pI) were 5.22-8.19 (Table 1). With the exception of BjuO006283 contig, which could not be anchored in the chromosome of B. juncea var. tumida, the left 17 BjuTIR1/AFBs genes located are in 11 of 20 B. juncea chromosomes. Each of the chromosome A03, A09, and B06 contains three genes, and the other eight genes were located at different chromosomes (Figure 1). BjuTIR1A, BjuTIR1D, and BjuTIR1F were located at the same chromosome of B06 (Figure 1).

Table 1. The transport inhibitor response 1/auxin signaling f-box protein (TIR1/AFB) family members in Brassica juncea var. tumida.

\begin{tabular}{|c|c|c|c|c|c|c|c|c|c|}
\hline Group & Gene Name & Locus & Sequence ID & Exon & Genomics (bp) & CDS (bp) & Protein (aa) & pI & MW (kD) \\
\hline \multirow[t]{6}{*}{ TIR1 } & BjuTIR1A & B06 & BjuB022928 & 3 & 2360 & 1788 & 595 & 6.80 & 66.87 \\
\hline & BjuTIR1B & A09 & BjuA036398 & 3 & 2340 & 1785 & 594 & 7.42 & 66.75 \\
\hline & BjuTIR1C & $\mathrm{A} 07$ & BjuA026836 & 3 & 2318 & 1785 & 594 & 7.70 & 66.72 \\
\hline & BjuTIR1D & B06 & BjuB006272 & 3 & 2302 & 1785 & 594 & 7.96 & 66.86 \\
\hline & BjuTIR1E & A04 & BjuA014423 & 3 & 2428 & 1776 & 591 & 7.41 & 66.57 \\
\hline & BjuTIR1F & B06 & BjuB022182 & 3 & 2667 & 1566 & 521 & 8.13 & 58.58 \\
\hline \multirow[t]{4}{*}{ AFB1 } & BjuAFB1A & A09 & BjuA031507 & 3 & 2746 & 1755 & 584 & 8.14 & 66.24 \\
\hline & BjuAFB1B & A03 & BjuA042044 & 2 & 1873 & 1758 & 585 & 5.22 & 65.24 \\
\hline & BjuAFB1C & A03 & BjuA010943 & 3 & 2540 & 1758 & 585 & 5.49 & 65.52 \\
\hline & BjuAFB1D & B08 & BjuB018573 & 4 & 2489 & 1680 & 559 & 8.19 & 63.57 \\
\hline AFB2 & BjuAFB2 & B04 & BjuB027337 & 3 & 2230 & 1731 & 576 & 6.30 & 64.74 \\
\hline \multirow[t]{4}{*}{ AFB3 } & BjuAFB3A & A08 & BjuA031011 & 3 & 2266 & 1779 & 592 & 5.94 & 66.60 \\
\hline & BjuAFB3B & B03 & BjuB033204 & 3 & 1917 & 1773 & 590 & 6.13 & 66.41 \\
\hline & BjuAFB3C & A09 & BjuA036983 & 3 & 2269 & 1734 & 577 & 6.49 & 65.04 \\
\hline & BjuAFB3D & B02 & BjuB048292 & 4 & 2136 & 1659 & 552 & 5.68 & 62.07 \\
\hline AFB4 & BjuAFB4 & B05 & BjuB014241 & 3 & 1987 & 1830 & 609 & 5.58 & 68.13 \\
\hline \multirow[t]{2}{*}{ AFB5 } & BjuAFB5A & Contig & BjuO006283 & 3 & 2017 & 1818 & 605 & 5.52 & 67.81 \\
\hline & BjuAFB5B & A03 & BjuA011137 & 3 & 2167 & 1821 & 606 & 5.54 & 67.84 \\
\hline
\end{tabular}

pI: Isoelectric point; MW: molecular weight.
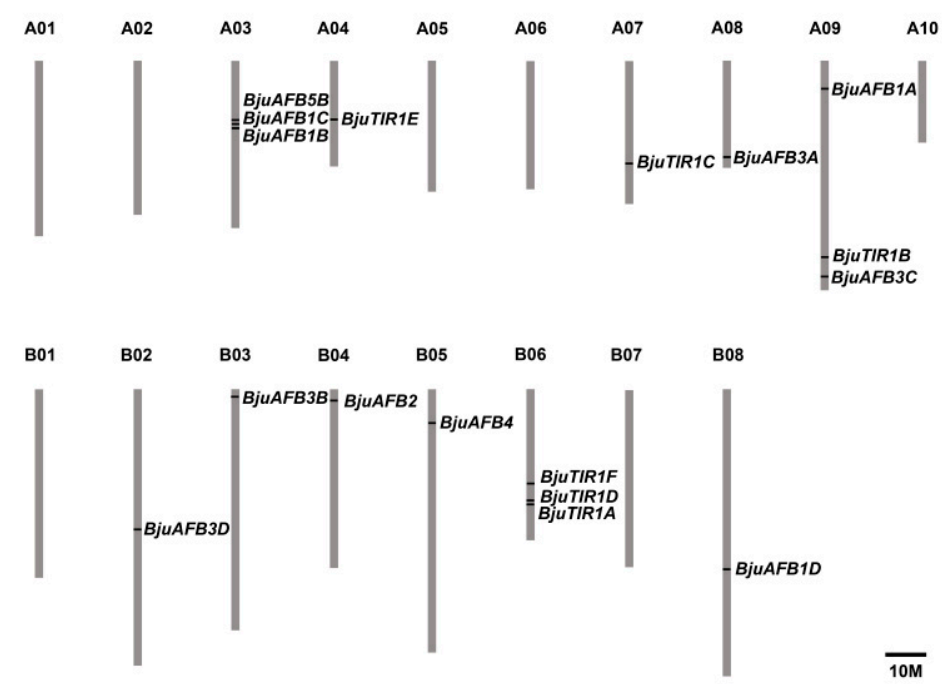

Figure 1. The gene locations of BjuTIR1 and BjuAFBs. Seventeen identified BjuTIR1/AFBs homologous genes were mapped to 11 out of 18 chromosomes. The chromosome name is at the top of each bar. The scale of the chromosome is in millions of bases $(\mathrm{Mb})$. 


\subsection{The Phylogenetic Analysis and Gene Structures of BjuTIR1/AFBs}

The TIR1/AFBs protein sequences of A. thaliana and B. juncea var. tumida were used for phylogenetic analysis, and six clades were obtained in the neighbor-joining phylogenetic tree (Figure 2). According to the phylogenetic analysis, six TIR1 homologs were identified, and named BjuTIR1A to BjuTIR1F; four AFB1 homologs were identified, and named BjuAFB1A to BjuAFB1D; one AFB2 homolog was identified, and named BjuAFB2; four AFB3 homologs were identified, and named BjuAFB3A to BjuAFB3D; one AFB4 homolog was identified, and named BjuAFB4; and two AFB5 homologs were identified, and named BjuAFB5A to BjAFB5B (Figure 2, Table 1). We also analyzed the phylogenic relationships of TIR1/AFB genes between B. juncea var. tumida and Brassica rapa, and the results showed that there was high sequence similarity between those genes (Figure S3). Interestingly, many TIR1/AFB genes are located at same chromosomes in each genome, respectively. For example, BjuTIR1C had highest sequence identity with Bra003518, and they were both located at chromosome A07 in their genome, respectively (Figure S3). According to gene structure analysis, most of the BjuTIR1/AFBs genes had similar gene structure to Arabidopsis TIR1/AFBs, which includes three exons and two introns. The exceptions were BjuAFB1D and BjuAFB3D, both of which contain four exons and three introns, as well as BjuAFB1B with two exons and one intron (Figure 2).

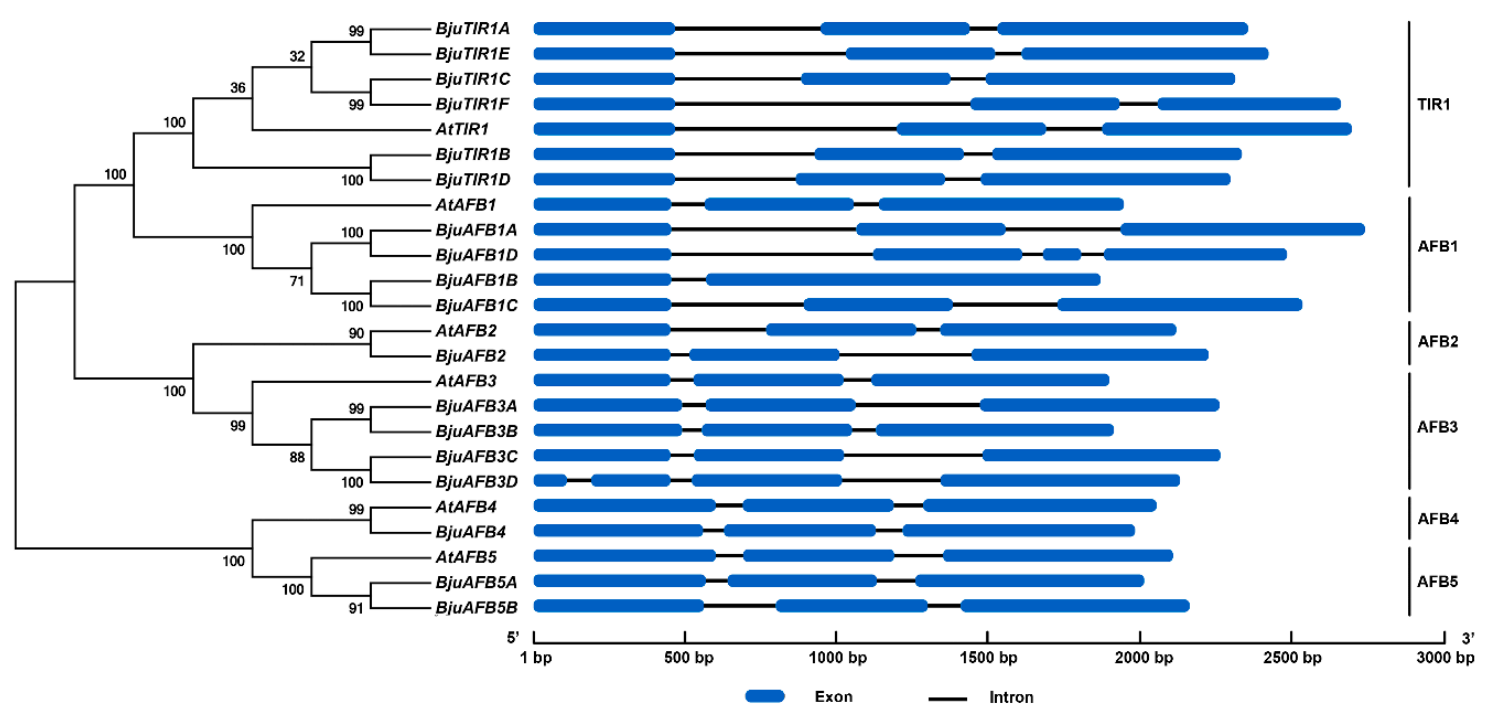

Figure 2. The phylogenetic analysis and gene structure of TIR1/AFBs in Arabidopsis thaliana and Brassica juncea. The protein sequence of each gene was used for the alignment, and the phylogenic neighbor-joining tree was constructed using MEGA5 phylogenetic analysis software.

\subsection{The Alignment of BjuTIR1/AFB Proteins and Secondary Domain Prediction}

The BjuTIR1/AFBs protein sequences were aligned by Clustalx [28], and the results showed that the sequences of those proteins were conserved (Figure 3). The sequence identification was more than $83.67 \%$ among the BjuTIR1 proteins. Similar results were found for BjuAFBs proteins: there was more than $90.77 \%, 89.21 \%$, and $98.36 \%$ sequence identification among BjuAFB1, BjuAFB3, and BjuAFB5 family proteins, respectively. In particular, the peptide sequences were highly conserved in the F-box domain region (Figure 3). 

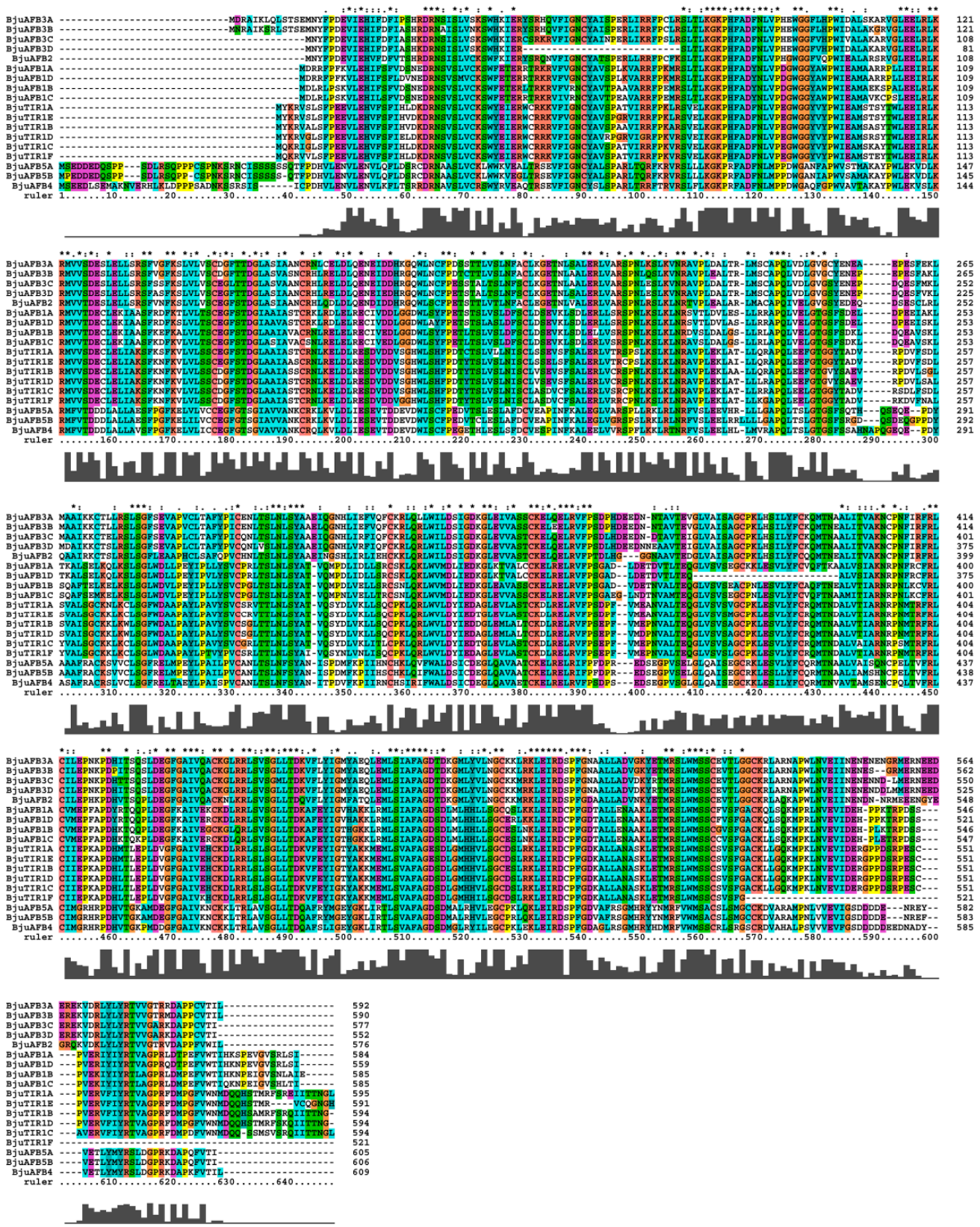

Figure 3. The protein sequence alignment of BjuTIR1/AFBs.

The secondary domains of BjuTIR1 and BjuAFBs were predicted, the results showed that all the BjuTIR1, BjuAFB1, BjuAFB2, and BjuAFB3 proteins contained an F-box domain in the N-terminus, indicating that those genes might function as E3 ubiquitin ligases. However, no F-box domains were found in the BjuAFB4 and BjuAFB5 proteins, suggesting that their genes function in a different manner. There were several LRR domains in all the BujTIR1 and BjuAFB proteins; those LRR domains were related to the interaction between the BjuTIR1/AFBs with other proteins (Figure 4). 


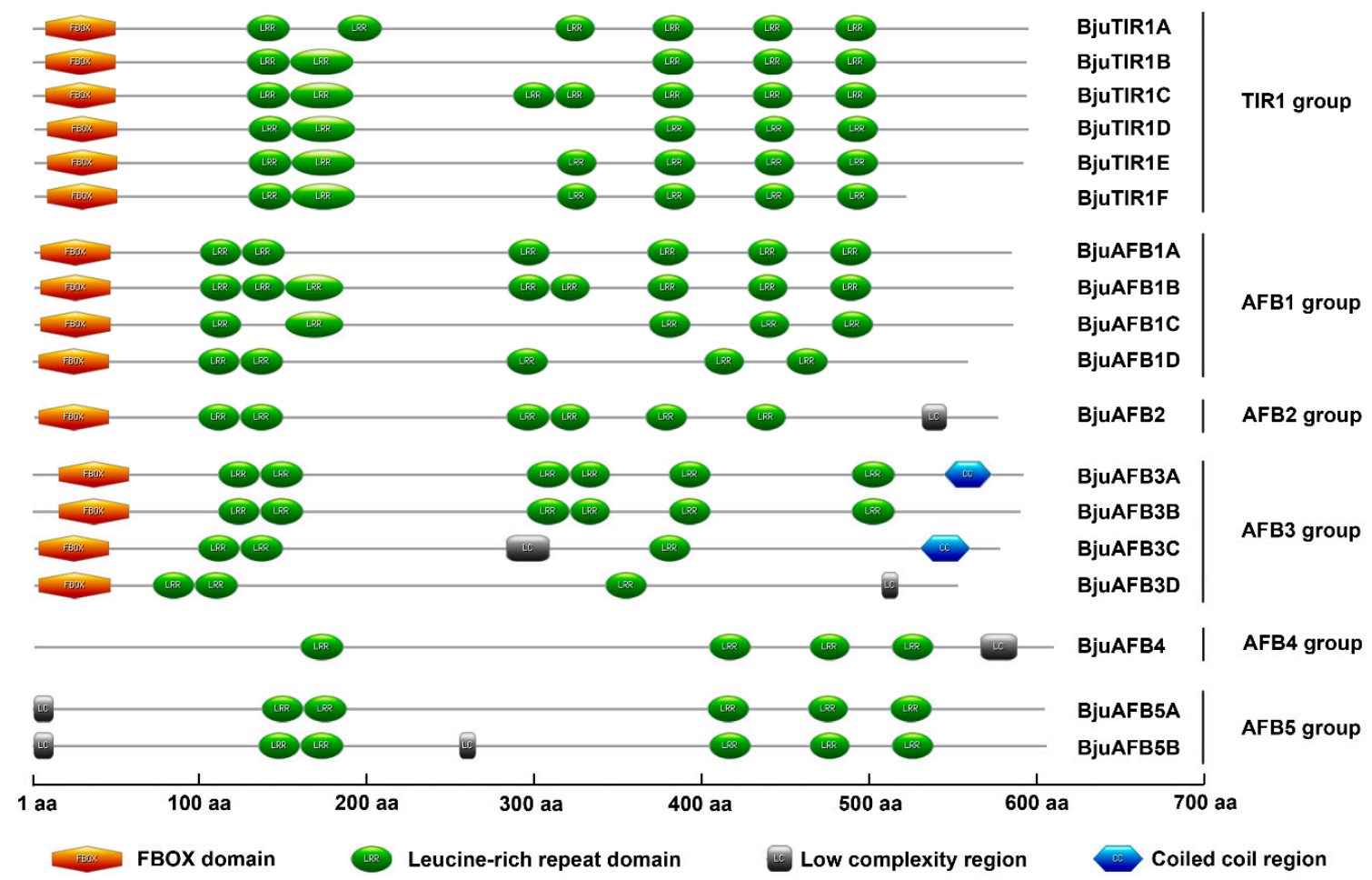

Figure 4. The secondary domains of BjuTIR1 and BjuAFB proteins.

\subsection{The Cis-Element Prediction of BjuTIR1/AFB Promoters}

The $2 \mathrm{~kb}$ DNA region upstream BjuTIR1/AFB sequences were chosen as promoters of those genes, and cis-elements were predicted by the PLACE software. Some auxin-responsive elements were found in most of BjuTIR1/AFBs' promoters, such as ARFAT (TGTCTC) [29] and GMSAUR (CATATG) [30] (Figure 5). There were also some cis-elements related to plant response to biotic-stresses, such as GT1GMSCAM4 (GAAAAA) [31] and GCCCORE (GCCGCC) [32]. The cis-elements MYCCONSENSUSAT (CANNTG) [33], MYB1AT (WAACCA) [33], and MYBATRD22 (CTAACCA) [34] were also found in the promoter region of most BjuTIR1/AFBs genes. Those cis-elements were involved in plant response to dehydration stress. Some cis-elements responsive to low temperatures were also found, such as LTRE1HVBLT49 (CCGAAA) [35], CRTDREHVCBF2 (GTCGAC) [36], and LTRECOREATCOR15 (CCGAC) [37]. Besides that, some cis-elements responsive to ABA (ABRELATERD1 [38] and ABREATRE22 [39]) and salicylic acid (SA) (ASF1MOTIFCAMV) [40] were also included in the promoter regions of those genes (Figure 5). Together, several cis-elements responsive to auxin, $\mathrm{ABA}, \mathrm{SA}$, biotic stresses, and abiotic stresses existed in the promoter of BjuTIR1 and BjuAFBs family genes. We also analyzed those cis-elements in the promoters of AtTIR1/AFB genes. The results showed that most of the cis-elements could be found in the promoters of Arabidopsis TIR1/AFB genes, except GMSAUR, GCCCORE, CRTDREHVCBF2, and ABREATRE22 (Figure 5). This result suggests that those four cis-elements might specifically play roles in B. juncea var. tumida. 


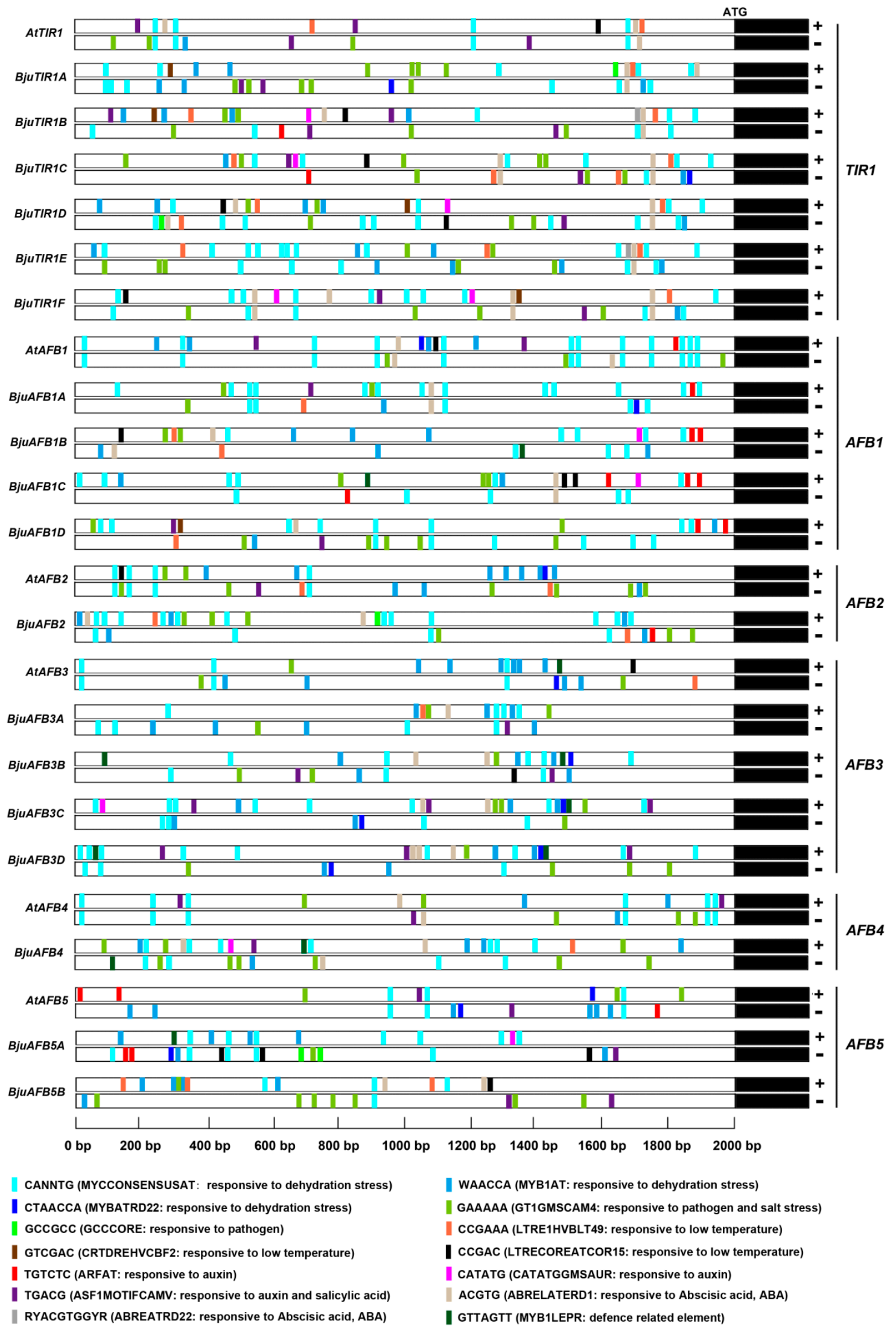

Figure 5. The cis-element prediction of BjuTIR1/AFBs promoters. The promoter sequences (2000 bp) upstream of the genes were chosen for cis-elements analysis using the software at http:/ /www.dna. affrc.go.jp/PLACE/. 


\subsection{The Transcriptional Expression Pattern of BjuTIR1/AFBs in Different Tissue}

The seedlings of Yong An Xiao Ye were cultivated in a field for six months, and the root, stem, swollen stem, leaf, flower, and pod were harvested for RNA extraction. The real-time quantitative PCR were performed to detect the expression pattern of BjTIR1/AFB family genes; the raw data of the qPCR results can be found in the Supplementary Materials (File S4). The results showed that BjuTIR1A, $B j u T I R 1 D$, and BjuAFB1B were highly expressed in most tissues, and moderate expression levels were found for BjuTIR1C, BjuAFB1B, BjuAFB3A, and BjuAFB3C (Figure 6). BjuAFB1D, BjuAFB2, BjuAFB3D, and $B j u A F B 5 A$ demonstrated lower expression levels in all tissues, suggesting that those four genes had limited function during plant growth and development (Figure 6). As shown in Figure 6, BjuTIR1A was expressed at much higher levels in the flower than in any other tissues, indicating this gene might be involved in flower development. Many genes, such as BjuTIR1B, BjuTIR1C, BjuTIR1D, BjuTIR1F, $B j u A F B 1 B$ and $A F B 3 C$, showed their highest expression levels in pods, suggesting that those genes might be responsible for pod formation. All the BjuTIR1 genes were highly expressed in the pod except BjuTIR1A, which indicates that BjuTIR1 family genes mainly function in pod formation and development (Figure 6). Besides that, the high expression of BjuTIR1A, BjuTIR1D, BjuAFB1B, and $A F B 3 A$ in the stem as well as the observed swollen stem suggests that those genes might play important roles in the function of stem development (Figure 6).

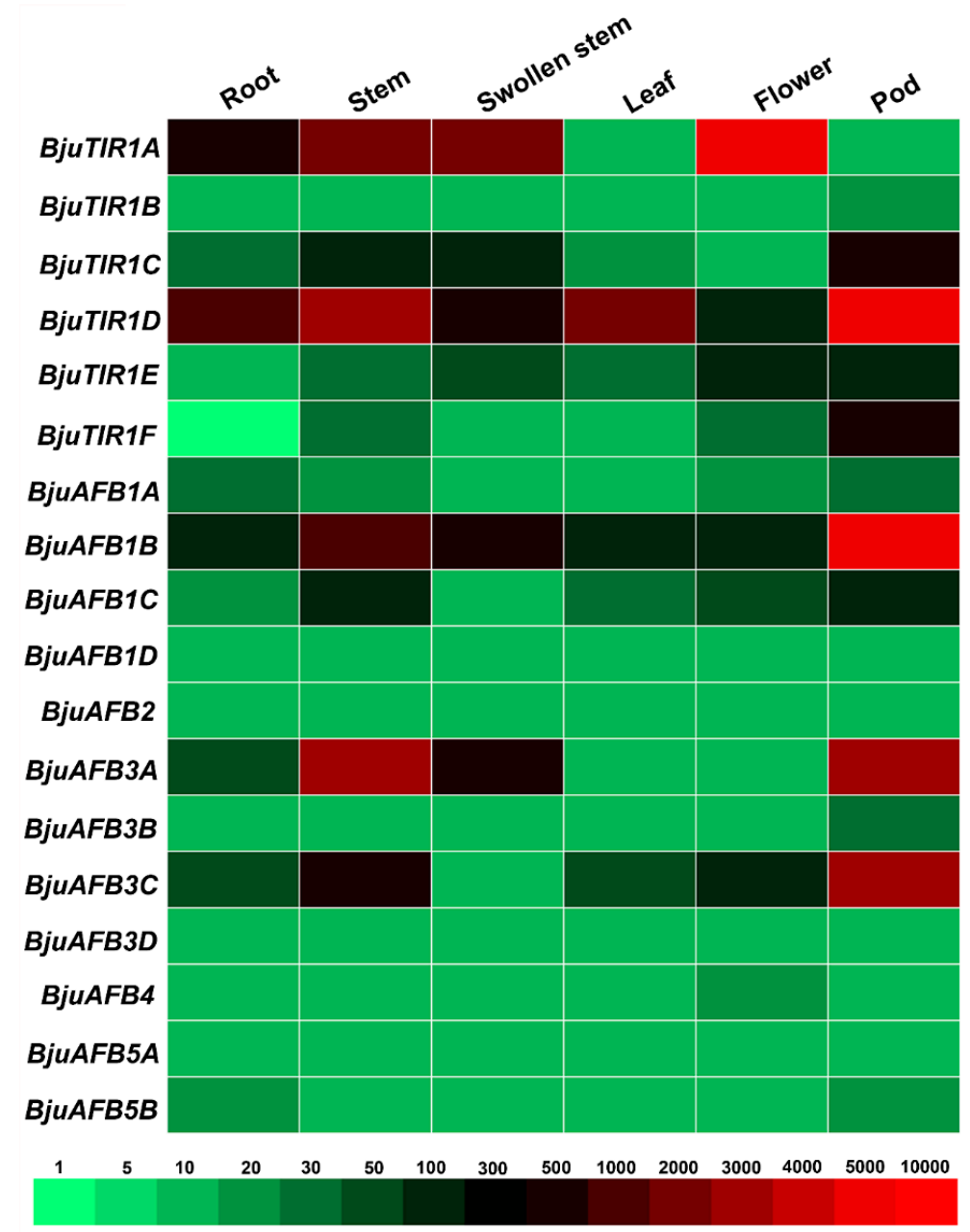

Figure 6. The expression patterns of BjuTIR1/AFB genes in different tissues. The tissue-specific expression pattern of BjuTIR1/AFB homologous genes were analyzed by qPCR. BjuActin3 was used as the internal control. The boxes displayed the gene expression levels using the $2^{-\Delta \Delta C T}$ method, and different colors represent different expression levels under the marked threshold values above the ruler. 


\subsection{The Transcriptional Expression Pattern of BjuTIR1/AFBs Under Salt Stress Treatment}

To investigate candidate BjuTIR1/AFB genes' interaction with plant response to salt stress, the gene expression levels were tested. Two-week-old seedlings of Yong An Xiao Ye were treated by $200 \mathrm{mM}$ $\mathrm{NaCl}$ for 3, 6, 12, and $24 \mathrm{~h}$, respectively, and the roots were collected for RT-qPCR analysis. The results showed that BjuTIR1A was significantly induced by salt treatment, especially after 3 and $6 \mathrm{~h}$ treatment, as the transcriptional expression levels of BjuTIR1B and BjuAFB1C increased at 3 and $6 \mathrm{~h}$, respectively (Figure 7). In contrast, the expression of most $B j u T I R 1 / A F B$ family genes were inhibited by salt treatment, including BjuTIR1C, BjuTIR1D, BjuTIR1E, BjuAFB1A, BjuAFB1B, BjuAFB3A, BjuAFB3B, $B j u A F B 3 C$, and BjuAFB4 (Figure 7). Those results suggest that most of the BjuTIR1/AFB genes were repressed by salt treatment, and might be involved in plant responses to salt stress.
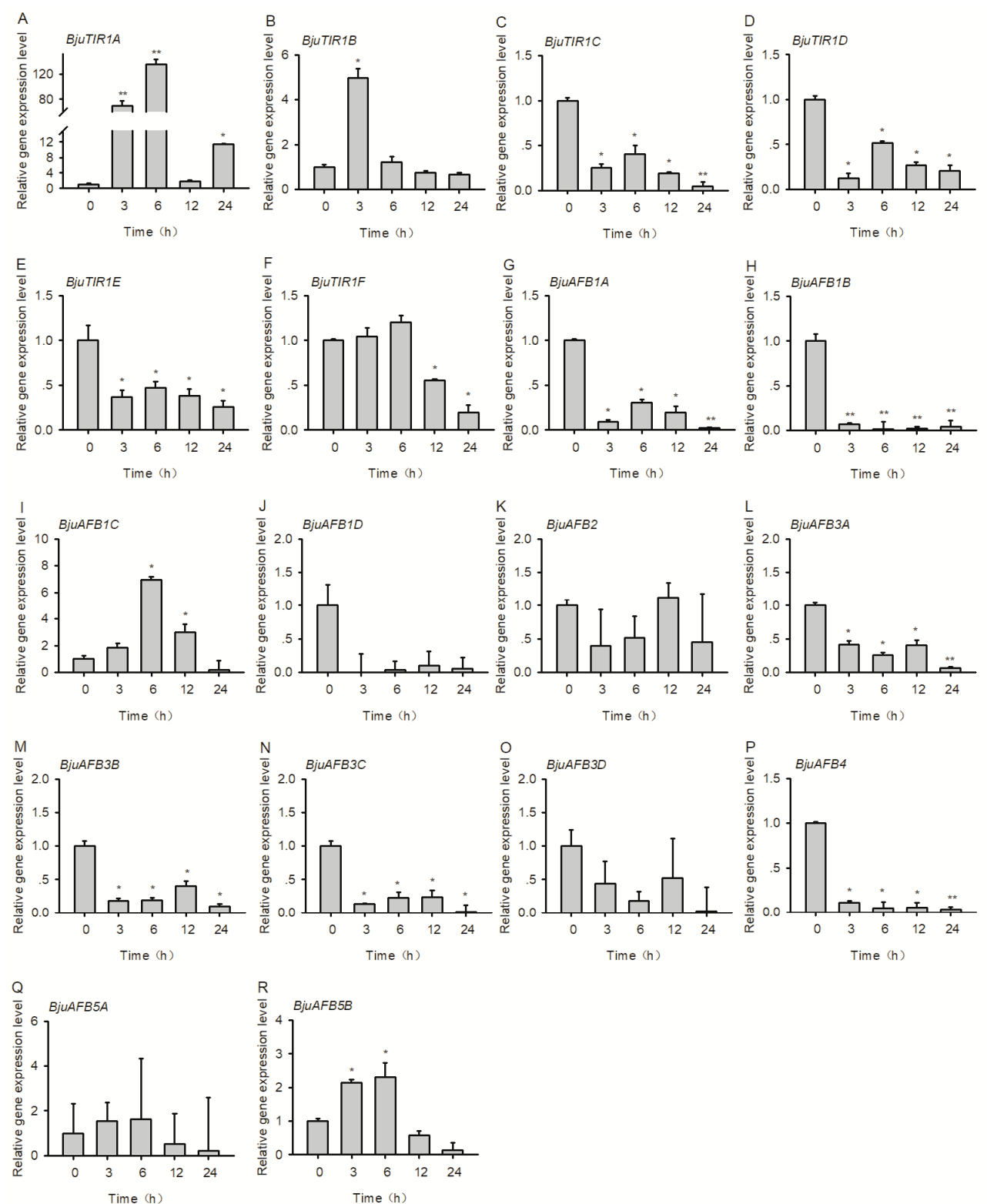

Figure 7. The expression patterns of BjuTIR1/AFB genes under salt stress. The expression pattern of BjuTIR1/AFB homologous genes were analyzed by qPCR. BjuActin3 was used as the internal control. Three independent biological repeats were performed, and all data points were the means of three biological replicates \pm standard error (SE). Significant differences: ${ }^{*}, p<0.05 ;{ }^{* *}, p<0.01$. 
3.7. The Transcriptional Expression Pattern of BjuTIR1/AFBs under Plasmodiophora brassicae Treatment

Plasmodiophora brassicae is a main pathogen of B. juncea var. tumida, which could lead to club root of cruciferous plants. To test which gene may be involved in the plant response to pathogen attack, the expression patterns of BjuTIR1/AFB family genes were detected under P. brassicae treatment. Two-week-old seedlings of Yong An Xiao Ye were treated by P. brassicae $\left(\mathrm{OD}_{600}=0.07\right)$ for $6,12,24$, 48 , and $72 \mathrm{~h}$, respectively, and the roots were collected for (RT-qPCR) analysis. The results showed that BjuTIR1A and BjuAFB3B were severely induced by P. brassicae treatment; BjuTIR1D, BjuTIR1E, $B j u A F B 1 A, B j u A F B 1 B, B j u A F B 2, B j u A F B 3 D, B j u A F B 4$, and BjuAFB5B were induced moderately by P. brassicae treatment (Figure 8 ). Other genes showed similar expression levels during P. brassicae treatment, and nearly no gene was inhibited by this biotic stress treatment. Experiment results show the BjuTIR1/AFB family genes induced by $P$. brassicae could be involved in plant response to this pathogen—especially BjuTIR1A (Figure 8).
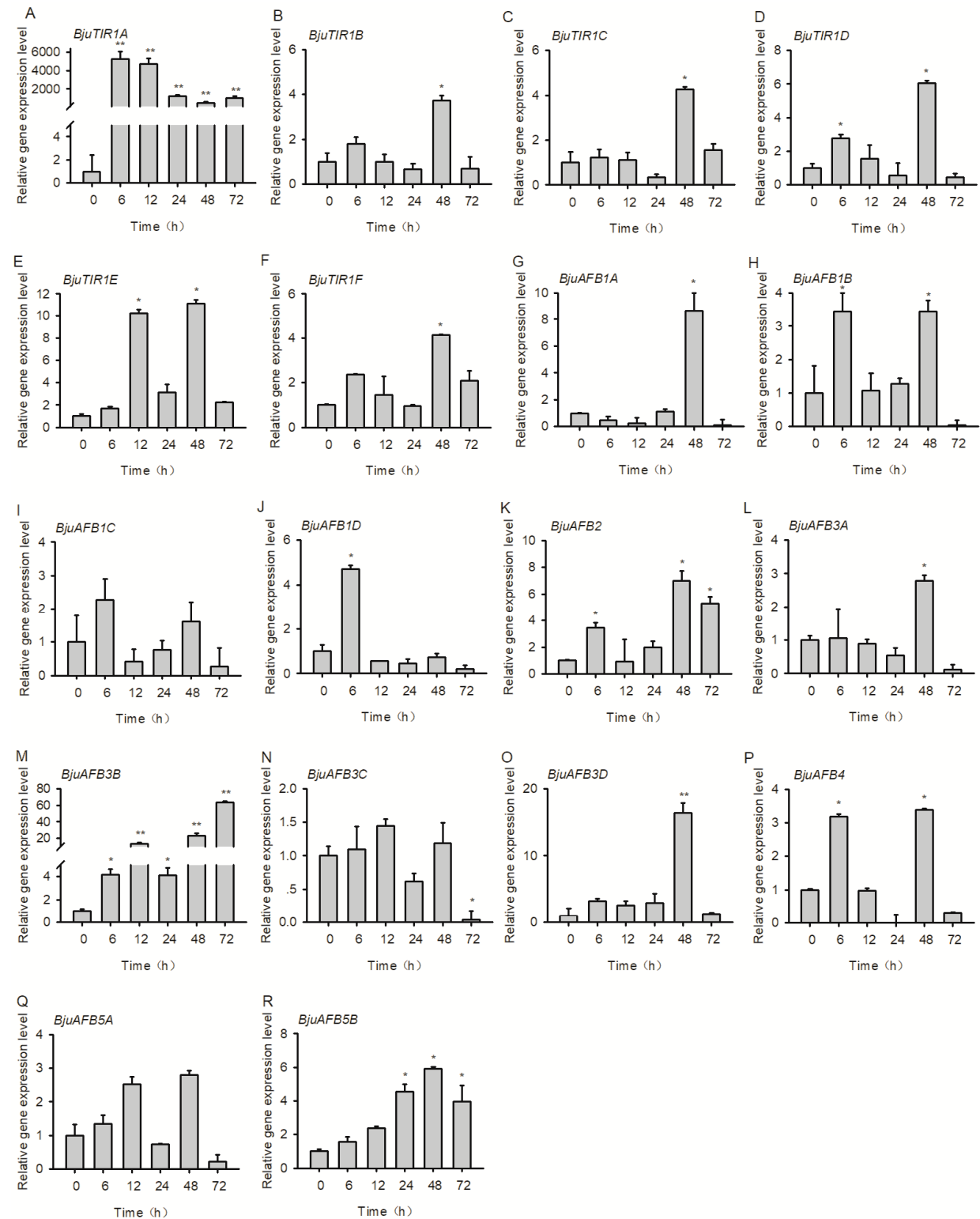

Figure 8. The expression patterns of BjuTIR1/AFB genes under the pathogen Plasmodiophora brassicae. The expression pattern of BjuTIR1/AFB homologous genes were analyzed by qPCR. BjuActin3 was used as the internal control. Three independent biological repeats were performed, and all data points were the means of three biological replicates \pm SE. Significant differences: ${ }^{*}, p<0.05 ;{ }^{* *}, p<0.01$. 


\section{Discussion}

B. juncea var. tumida is an allotetraploid species formed by hybridization between the diploid ancestors of B. rapa and Brassica nigra, followed by genome duplication [41]. In A. thaliana, six auxin receptor genes were identified [5,6,16-18]. According to our results, 18 putative auxin receptors were found in the genome of B. juncea var. tumida. Except for AtAFB2 and AtAFB4, each Arabidopsis auxin receptor was homologous to 2-6 genes in the $B$. juncea var. tumida genome (Table 1). The multiple homologs of auxin receptors in $B$. juncea var. tumida may be from the result of genome duplication. Among the 18 auxin receptors, nine genes were located in the A sub-genome and eight in the $\mathrm{B}$ sub-genome (BjuAFB5A could not be mapped to a genome). The comparable auxin receptor gene numbers in the A or B sub-genome indicates that the genome of $B$. juncea var. tumida experienced co-linearity in the gene duplication process. Most of the auxin receptor genes contained three exons and two introns, the exceptions being BjuAFB1D and BjuAFB1D, both of which contained four exons and three introns (Figure 2). The gene structure showed that more exons attributed to a partial exon coding sequence converted to a non-coding sequence. This exon-intron splicing arrangement is present in other species, such as rice and maize [42,43].

Abnormal phenotypes of enlarged flag leaf inclination, more tillers, early flowering and a lower tolerance to salt were found due to reduction of OsTIR1 and OsAFB2 in rice [44,45]. However, the expression levels of BjuAFB2 was low in all tissues of B. juncea var. tumida (Figure 8), suggesting that BjuAFB2 might not play an important role as a main auxin receptor. In contrast, the high expression levels of BjuTIR1A and BjuTIR1D indicate that these two genes might be the main auxin receptors in B. juncea var. tumida. According to the results of the gene expression pattern, tissue-specific gene expressions were identified, such as BjuTIR1A demonstrating influence on the regulation of flower development, and BjuTIR1F may specifically influence pod development regulation.

It has been reported that auxin signaling plays an important role in plant resistance to abiotic stress, especially at high salinity [3]. In this study, the expression levels of most of auxin receptor genes was inhibited under salt treatment (Figure 7). In tobacco, overexpression of AtmiR393a could repress the expression levels of auxin receptors like TIR1, leading to the inhibition of plant growth and enhanced resistance to salt stress [46]. The reduced expressions of BjuTIR1/AFB family genes under salt treatment were consistent with the previous study. The exception was BjuTIR1A-the expression of this gene was significantly induced by salt treatment, which might result from feedback of the signaling transduction.

The auxin signaling pathway mediated by TIR1/AFBs is usually involved in plant resistance or tolerance to pathogen attacks, such the anthracnose disease in cassava [47], Zea mays defense against Rhizoctonia solani [48], or Arabidopsis resistance to the bacterium Pseudomonas syringae [25]. In Arabidopsis, AtTIR1 and AtAFB1 are transcriptionally upregulated in clubroots, and the mutants of tir1, afb1-3, and afb1-3 afb2-3 all resulted in more susceptible reactions to the root pathogen P. brassicae [49]. In this study, similar results were found. The transcriptional level of BjuTIR1A was severely induced by P. brassicae treatment, especially at 6 and $12 \mathrm{~h}$ after inoculation. Besides that, all the BjuTIR1s were induced by this pathogen treatment, except for BjuTIR1B (Figure 8). For BjuAFB1 genes, the expression levels of $A F B 1 A, A F B 1 B$, and $A F B 1 C$ also increased at $48 \mathrm{~h}$ after pathogen inoculation (Figure 8). The induction of those genes suggested that BjuTIR1s and BjuAFB1s might play similar roles to AtTIR1/AFB1s in the processes of plant defense against $P$. brassicae invasion.

\section{Conclusions}

In this study, a total of 18 BjuTIR1/AFB genes were identified, and they have similar gene structures and protein domains to the auxin receptors of Arabidopsis. Several cis-elements related to plant response to phytohormones, biotic stresses, and abiotic stresses were found in the promoter of BjuTIR1/AFB genes, indicating those genes may play a role in the regulation of plant development and defense against biotic and abiotic stresses, which could benefit from a more targeted follow-up study. Gene expression analysis showed that some BjuTIR1/AFB family genes exhibit a special expression pattern, 
and most genes are responsive to salt stress treatment. Together, our data provides a useful foundation for future research regarding the function of auxin receptors in B. juncea var. tumida.

Supplementary Materials: The following are available online at http:/ /www.mdpi.com/2073-4425/10/2/165/s1, Table S1: Primers used for qPCR in this study; File S2: The CDS sequences of TIR1/AFBs; Figure S3: The phylogenic analysis of TIR1/AFBs in A. thaliana, B. rapa and B. juncea var. tumida. The protein sequence of each gene was used for the alignment, and the phylogenic neighbor-joining tree was constructed using MEGA5 phylogenetic analysis software. File S4: The raw data of qPCR assays.

Author Contributions: D.W. and Z.C. conceived the project and designed the experiments. Z.C., D.-e.Z., J.L., C.C., Z.S., M.X., M.F., and Y.C. performed the experiments and analyzed the data; D.W. and Z.C. prepared the manuscript.

Funding: This work was supported by the National Natural Science Foundation of China (Program Nos. 31701451, 31770407 and 31800311), the Chongqing Basic Research and Frontier Exploration Project (Program No. cstc2018jcyjAX0628), the Science and Technology Research Program of Chongqing Municipal Education Commission (Grant No. KJQN201801434), and the Yangtze Normal University Startup Foundation for Scientific Research (Program Nos. 2017KYQD151 and 2017KYQD152).

Conflicts of Interest: The authors declare that they have no competing interests for this research.

\section{References}

1. Kepinski, S.; Leyser, O. Plant development: Auxin in loops. Curr. Biol. 2005, 15, R208-R210. [CrossRef] [PubMed]

2. Perrot-Rechenmann, C. Cellular responses to auxin: Division versus expansion. CSH Perspect. Biol. 2010, 2, a001446. [CrossRef] [PubMed]

3. Iglesias, M.J.; Terrile, M.C.; Windels, D.; Lombardo, M.C.; Bartoli, C.G.; Vazquez, F.; Estelle, M.; Casalongué, C.A. MiR393 regulation of auxin signaling and redox-related components during acclimation to salinity in Arabidopsis. PLoS ONE 2014, 9, e107678. [CrossRef] [PubMed]

4. Takatsuka, H.; Umeda, M. Hormonal control of cell division and elongation along differentiation trajectories in roots. J. Exp. Bot. 2014, 65, 2633-2643. [CrossRef] [PubMed]

5. Dharmasiri, N.; Dharmasiri, S.; Weijers, D.; Lechner, E.; Yamada, M.; Hobbie, L.; Ehrismann, J.S.; Jürgens, G.; Estelle, M. Plant development is regulated by a family of auxin receptor F box proteins. Dev. Cell 2005, 9, 109-119. [CrossRef] [PubMed]

6. Kepinski, S.; Leyser, O. The Arabidopsis F-box protein TIR1 is an auxin receptor. Nature 2005, 435, 446-451. [CrossRef] [PubMed]

7. Cai, Z.; Wang, Y.; Zhu, L.; Tian, Y.; Chen, L.; Sun, Z.; Ullah, I.; Li, X. GmTIR1/GmAFB3-based auxin perception regulated by miR393 modulates soybean nodulation. New Phytol. 2017, 215, 672-686. [CrossRef] [PubMed]

8. Dharmasiri, N.; Dharmasiri, S.; Jones, A.M.; Estelle, M. Auxin action in a cell-free system. Curr. Biol. 2003, 13, 1418-1422. [CrossRef]

9. Wang, R.; Estelle, M. Diversity and specificity: Auxin perception and signaling through the TIR1/AFB pathway. Curr. Opin. Plant Biol. 2014, 21, 51-58. [CrossRef] [PubMed]

10. Salehin, M.; Bagchi, R.; Estelle, M. SCFTIR1/AFB-based auxin perception: Mechanism and role in plant growth and development. Plant Cell 2015, 27, 9-19. [CrossRef] [PubMed]

11. Lavy, M.; Estelle, M. Mechanisms of auxin signaling. Development 2016, 143, 3226-3229. [CrossRef] [PubMed]

12. Benjamins, R.; Scheres, B. Auxin: The looping star in plant development. Annu. Rev. Plant Biol. 2018, 59, 443-465. [CrossRef] [PubMed]

13. Ruegger, M.; Dewey, E.; Gray, W.M.; Hobbie, L.; Turner, J.; Estelle, M. The TIR1 protein of Arabidopsis functions in auxin response and is related to human SKP2 and yeast grr1p. Genes Dev. 1998, 12, 198-207. [CrossRef] [PubMed]

14. Gray, W.M.; del Pozo, J.C.; Walker, L.; Hobbie, L.; Risseeuw, E.; Banks, T.; Crosby, W.L.; Yang, M.; Ma, H.; Estelle, M. Identification of an SCF ubiquitin-ligase complex required for auxin response in Arabidopsis thaliana. Genes Dev. 1999, 13, 1678-1691. [CrossRef] [PubMed]

15. Yu, H.; Zhang, Y.; Moss, B.L.; Bargmann, B.O.R.; Wang, R.; Prigge, M.; Nemhauser, J.L.; Estelle, M. Untethering the TIR1 auxin receptor from the SCF complex increases its stability and inhibits auxin response. Nat. Plants 2015, 1, 14030. [CrossRef] [PubMed] 
16. Prigge, M.; Greenham, K.; Zhang, Y.; Santner, A.; Castillejo, C.; Mutka, A.M.; O'Malley, R.C.; Ecker, J.R.; Kunkel, B.N.; Estelle, M. The Arabidopsis auxin receptor F-box proteins AFB4 and AFB5 are required for response to the synthetic auxin picloram. G3-Genes Genom. Genet. 2016, 6, 1383-1390. [CrossRef] [PubMed]

17. Walsh, T.A.; Neal, R.; Merlo, A.O.; Honma, M.; Hicks, G.R.; Wolff, K.; Matsumura, W.; Davies, J.P. Mutations in an auxin receptor homolog AFB5 and in SGT1B confer resistance to synthetic picolinate auxins and not to 2,4-dichlorophenoxyacetic acid or indole-3-acetic acid in Arabidopsis. Plant Physiol. 2006, 142, 542-552. [CrossRef] [PubMed]

18. Calderón Villalobos, L.I.; Lee, S.; De Oliveira, C.; Ivetac, A.; Brandt, W.; Armitage, L.; Sheard, L.B.; Tan, X.; Parry, G.; Mao, H.; et al. A combinatorial TIR1/AFB-Aux/IAA co-receptor system for differential sensing of auxin. Nat. Chem. Biol. 2016, 8, 477-485. [CrossRef] [PubMed]

19. Scheitz, K.; Lüthen, H.; Schenck, D. Rapid auxin-induced root growth inhibition requires the TIR and AFB: Auxin receptors. Planta 2013, 238, 1171-1176. [CrossRef] [PubMed]

20. Fendrych, M.; Akhmanova, M.; Merrin, J.; Glanc, M.; Hagihara, S.; Takahashi, K.; Uchida, N.; Torii, K.U.; Friml, J. Rapid and reversible root growth inhibition by TIR1 auxin signalling. Nat. Plants 2018, 4, 453-459. [CrossRef] [PubMed]

21. Chen, Z.; Bao, M.; Sun, Y.; Yang, Y.; Xu, X.; Wang, J.; Han, N.; Bian, H.; Zhu, M. Regulation of auxin response by miR393-targeted transport inhibitor response protein 1 is involved in normal development in Arabidopsis. Plant Mol. Biol. 2011, 77, 619-629. [CrossRef] [PubMed]

22. Vidal, E.A.; Araus, V.; Lu, C.; Parry, G.; Green, P.J.; Coruzzi, G.M.; Gutiérrez, R.A. Nitrate-responsive miR393/AFB3 regulatory module controls root system architecture in Arabidopsis thaliana. Proc. Natl. Acad. Sci. USA 2010, 107, 4477-4482. [CrossRef] [PubMed]

23. Hu, Z.; Keçeli, M.A.; Piisilä, M.; Li, J.; Survila, M.; Heino, P.; Brader, G.; Palva, E.T.; Li, J. F-box protein AFB4 plays a crucial role in plant growth, development and innate immunity. Cell Res. 2012, 22, 777-781. [CrossRef] [PubMed]

24. Chen, H.; Li, Z.; Xiong, L. A plant microRNA regulates the adaptation of roots to drought stress. FEBS Lett. 2012, 586, 1742-1747. [CrossRef] [PubMed]

25. Navarro, L.; Dunoyer, P.; Jay, F.; Arnold, B.; Dharmasiri, N.; Estelle, M.; Voinnet, O.; Jones, J.D.G. A plant miRNA contributes to antibacterial resistance by repressing auxin signaling. Science 2006, 312, 436-439. [CrossRef] [PubMed]

26. Tamura, K.; Peterson, D.; Peterson, N.; Stecher, G.; Nei, M.; Kumar, S. MEGA5: Molecular evolutionary genetics analysis using maximum likelihood, evolutionary distance, and maximum parsimony methods. Mol. Biol. Evol. 2011, 28, 2731-2739. [CrossRef] [PubMed]

27. Chen, J.; Piao, Y.; Liu, Y.; Li, X.; Piao, Z. Genome-wide identification and expression analysis of chitinase gene family in Brassica rapa reveals its role in clubroot resistance. Plant Sci. 2018, 270, 257-267. [CrossRef] [PubMed]

28. Thompson, J.D.; Gibson, T.J.; Higgins, D.G. Multiple sequence alignment using ClustalW and ClustalX. Curr. Protoc. Bioinform. 2002, 2.3.1-2.3.22, Chapter 2(Unit 2), Unit 2.3. [CrossRef] [PubMed]

29. Nag, R.; Maity, M.K.; Dasgupta, M. Dual DNA binding property of ABA insensitive 3 like factors targeted to promoters responsive to ABA and auxin. Plant Mol. Biol. 2005, 59, 821-838. [CrossRef] [PubMed]

30. Xu, N.; Hagen, G.; Guilfoyle, T. Multiple auxin response modules in the soybean SAUR 15A promoter. Plant Sci. 1997, 126, 193-201. [CrossRef]

31. Park, H.C.; Kim, M.L.; Kang, Y.H.; Jeon, J.M.; Yoo, J.H.; Kim, M.C.; Park, C.Y.; Jeong, J.C.; Moon, B.C.; Lee, J.H.; et al. Pathogen- and NaCl-induced expression of the SCaM-4 promoter is mediated in part by a GT-1 box that interacts with a GT-1-like transcription factor. Plant Physiol. 2004, 135, 2150-2161. [CrossRef] [PubMed]

32. Brown, R.L.; Kazan, K.; McGrath, K.C.; Maclean, D.J.; Manners, J.M. A role for the GCC-box in jasmonate-mediated activation of the PDF1.2 gene of Arabidopsis. Plant Physiol. 2003, 132, 1020-1032. [CrossRef] [PubMed]

33. Abe, H.; Urao, T.; Ito, T.; Seki, M.; Shinozaki, K.; Yamaguchi-Shinozaki, K. Arabidopsis AtMYC2 (bHLH) and AtMYB2 (MYB) function as transcriptional activators in abscisic acid signaling. Plant Cell 2003, 15, 63-78. [CrossRef] [PubMed] 
34. Abe, H.; Yamaguchi-Shinozaki, k.; Urao, T.; Iwasaki, T.; Hosokawa, D.; Shinozaki, K. Role of Arabidopsis MYC and MYB homologs in drought- and abscisic acid-regulated gene expression. Plant Cell 1997, 9, 1859-1868. [PubMed]

35. Dunn, M.A.; White, A.J.; Vural, S.; Hughes, M.A. Identification of promoter elements in a low-temperature-responsive gene (blt4.9) from barley (Hordeum vulgare L.). Plant Mol. Biol. 1998, 38, 551-564. [CrossRef] [PubMed]

36. Xue, G.P. The DNA-binding activity of an AP2 transcriptional activator HvCBF2 involved in regulation of low-temperature responsive genes in barley is modulated by temperature. Plant J. 2003, 33, 373-383. [CrossRef] [PubMed]

37. Baker, S.S.; Wilhelm, K.S.; Thomashow, M.F. The 5'-region of Arabidopsis thaliana cor15a has cis-acting elements that confer cold-, drought- and ABA-regulated gene expression. Plant Mol. Biol. 1994, 24, 701-713. [CrossRef] [PubMed]

38. Simpson, S.D.; Nakashima, K.; Narusaka, Y.; Seki, M.; Shinozaki, K.; Yamaguchi-Shinozaki, K. Two different novel cis-acting elements of erd1, a clpA homologous Arabidopsis gene function in induction by dehydration stress and dark-induced senescence. Plant J. 2010, 33, 259-270. [CrossRef]

39. Iwasaki, T.; Yamaguchi-Shinozaki, K.; Shinozaki, K. Identification of a cis-regulatory region of a gene in Arabidopsis thaliana whose induction by dehydration is mediated by abscisic acid and requires protein synthesis. Mol. Gen. Genet. 1995, 247, 391-398. [CrossRef] [PubMed]

40. Redman, J.; Whitcraft, J.; Johnson, C.; Arias, J. Abiotic and biotic stress differentially stimulate as-1 element activity in Arabidopsis. Plant Cell Rep. 2002, 21, 180-185.

41. Yang, J.; Liu, D.; Wang, X.; Ji, C.; Cheng, F.; Liu, B.; Hu, Z.; Chen, S.; Pental, D.; Ju, Y.; et al. The genome sequence of allopolyploid Brassica juncea and analysis of differential homoeolog gene expression influencing selection. Nat. Genet. 2016, 48, 1225-1234. [CrossRef] [PubMed]

42. Wang, D.; Pei, K.; Fu, Y.; Sun, Z.; Li, S.; Liu, H.; Tang, K.; Han, B.; Tao, Y. Genome-wide analysis of the auxin response factors (ARF) gene family in rice (Oryza sativa). Gene 2007, 394, 0-24. [CrossRef] [PubMed]

43. Xing, H.; Pudake, R.N.; Guo, G.; Xing, G.; Hu, Z.; Zhang, Y.; Sun, Q.; Ni, Z. Genome-wide identification and expression profiling of auxin response factor $(A R F)$ gene family in maize. BMC Genom. 2011, 12, 178. [CrossRef] [PubMed]

44. Bian, H.; Xie, Y.; Guo, F.; Han, N.; Ma, S.; Zeng, Z.; Wang, J.; Yang, Y.; Zhu, M. Distinctive expression patterns and roles of the miRNA393/TIR1 homolog module in regulating flag leaf inclination and primary and crown root growth in rice (Oryza sativa). New Phytol. 2012, 196, 149-161. [CrossRef] [PubMed]

45. Xia, K.; Wang, R.; Ou, X.; Fang, Z.; Tian, C.; Duan, J.; Wang, Y.; Zhang, M. OsTIR1 and OsAFB2 downregulation via OsmiR393 overexpression leads to more tillers, early flowering and less tolerance to salt and drought in rice. PLoS ONE 2012, 7, e30039. [CrossRef] [PubMed]

46. Feng, X.; You, C.; Qiao, Y.; Mao, K.; Hao, Y. Ectopic overexpression of Arabidopsis AtmiR393a gene changes auxin sensitivity and enhances salt resistance in tobacco. Acta Physiol. Plant. 2010, 32, 997-1003. [CrossRef]

47. Pinweha, N.; Asvarak, T.; Viboonjun, U.; Narangajavana, J. Involvement of miR160/miR393 and their targets in cassava responses to anthracnose disease. J. Plant Physiol. 2015, 174, 26-35. [CrossRef] [PubMed]

48. Luo, M.; Gao, J.; Peng, H.; Pan, G.; Zhang, Z. MiR393-targeted TIR1-like (F-box) gene in response to inoculation to R. Solani in Zea mays. Acta Physiol. Plant. 2014, 36, 1283-1291. [CrossRef]

49. Jahn, L.; Mucha, S.; Bergmann, S.; Horn, C.; Staswick, P.; Steffens, B.; Siemens, J.; Ludwig-Müller, J. The clubroot pathogen (Plasmodiophora brassicae) influences auxin signaling to regulate auxin homeostasis in Arabidopsis. Plants 2013, 2, 726-749. [CrossRef] [PubMed]

(C) 2019 by the authors. Licensee MDPI, Basel, Switzerland. This article is an open access article distributed under the terms and conditions of the Creative Commons Attribution (CC BY) license (http:// creativecommons.org/licenses/by/4.0/). 